\title{
A Case of Brain Metastasis from Pulmonary Giant Cell Carcinoma
}

\author{
NAOSHI HAGIHARA, TOSHI ABE*, TOMIHIRO WAKAMIYA, YASUO SUGITA**, \\ MITSUO WATANABE AND KAZUO TABUCHI \\ Department of Neurosurgery, Koyanagi Memorial Hospital, Saga 840-2195 and \\ Departments of Radiology* and Pathology**, Kurume University School of Medicine, \\ Kurume 830-0011, Japan
}

Received 24 September 2009, accepted 22 January 2010

Edited by HISAMICHI AIZAWA

\begin{abstract}
Summary: A 74-year old female was admitted to our hospital due to sudden right hemiparesis. Precontrast brain computed tomography (CT) revealed multiple high-density masses consistent with multiple hemorrhage, and chest CT scan demonstrated a mass in the left lung field without hemorrhage. Follow-up CT showed enlargement of the multiple intracerebral hemorrhages. A diagnosis was made of brain metastasis from a rare pure giant cell carcinoma (GCC) of the lung.
\end{abstract}

Key words brain metastasis, pulmonary giant cell carcinoma, multiple hemorrhage

\section{INTRODUCTION}

The World Health Organization classification of lung carcinoma ranks the heterogeneous non-small cell lung cancer group, encompassing sarcoma or sarcoma-containing tumors under one heading: "carcinomas with pleomorphic, sarcomatoid or sarcomatous elements" [1]. This group contains entities such as pleomorphic carcinoma, spindle cell carcinoma, giant cell carcinoma (GCC), carcinosarcoma and pulmonary blastoma. These tumors are rare overall, making up approximately $0.1-0.4 \%$ of all lung malignancies [2].

\section{CASE REPORT}

A 74-year old female was admitted to our hospital due to sudden right hemiparesis. Neurological examination revealed mild hemiparesis of the right extremities. Precontrast brain computed tomography (CT) revealed multiple high-density masses consistent with multiple hemorrhage (Fig. 1). Magnetic resonance (MR) imaging detected all the hematoma at high sig- nal intensity because of bleeding accompanied by ring enhancement with Gadolinum. Contrast enhanced chest CT scan demonstrated a mass in the left lung field without hemorrhage (Fig. 2). Laboratory data showed mild elevation of LDH (282 IU/L), IL-2R $(538 \mathrm{U} / \mathrm{ml})$ and NSE $(52 \mathrm{ng} / \mathrm{ml})$. Bleeding and coagulation times were within normal range. After admission, the patient was kept in bed and monitored closely. Blood pressure remained within normal range without medication. At first, the cerebral hemorrhage was treated conservatively. She gradually became drowsy and right hemiparesis progressed. Follow-up CT, 7 days after admission, revealed enlargement of all hematoma. As a result, we performed evacuation of the hematomas and biopsy of the left frontal lobe lesion via a left frontal craniotomy. Histological examination identified the lesion as a pure GCC (Fig. 3). Immunohistochemically, cytokeratin AE1/E3 was positive, but CK7, CK20, thyroid transcription factor-1, and glial fibrillary acidic protein were negative. In addition to Hematoxilin-eosin staining, these immunostaining findings are consistent with GCC. The diagnosis was made of brain metasta-

Address for correspondence: Naoshi Hagihara, M.D., Department of Neurosurgery, Koyanagi Memorial Hospital, 230-2 Morodomitsu, Morodomi-cho, Saga city, Saga 840-2195, Japan. Tel: 81-952-47-3255 Fax: 81-952-47-5101 E-mail: hagihare167@ solid.ocn.ne.jp 


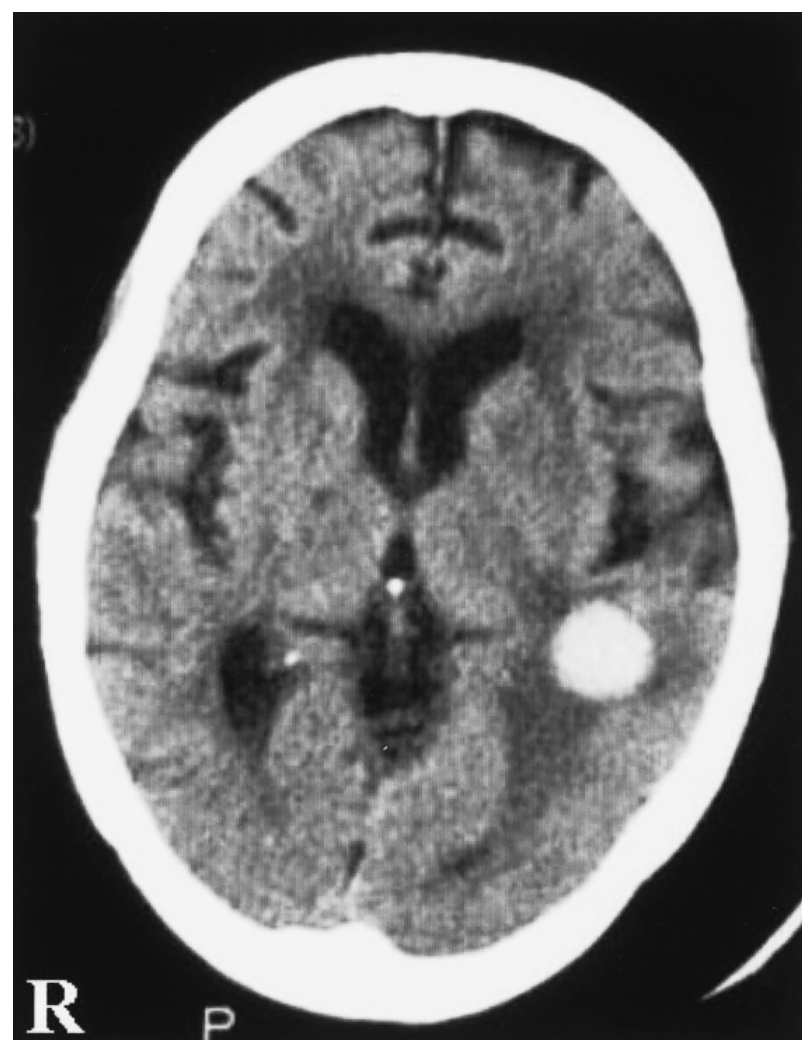

A

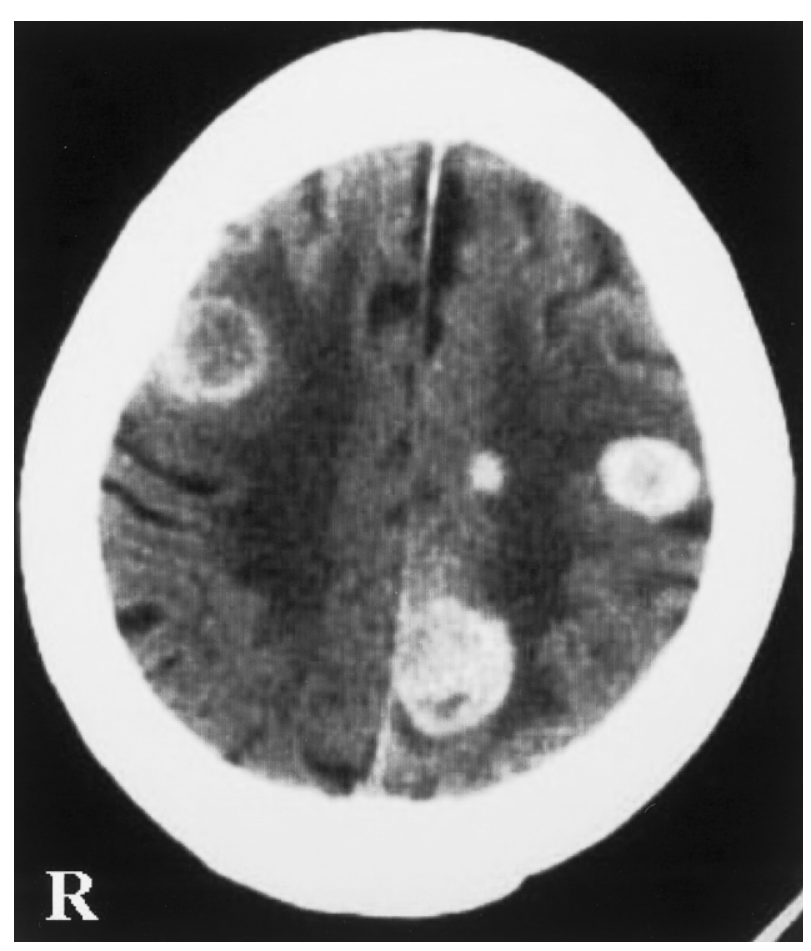

B

Fig. 1. Brain CT shows multiple hemorrhagic mass lesions in the cortico-medullary junction.

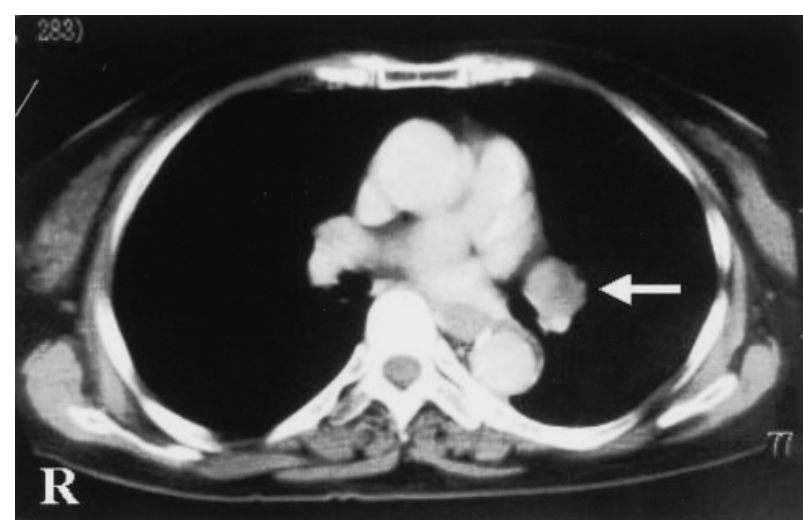

Fig. 2. Contrast enhanced chest CT showed pulmonary mass at left hilum.

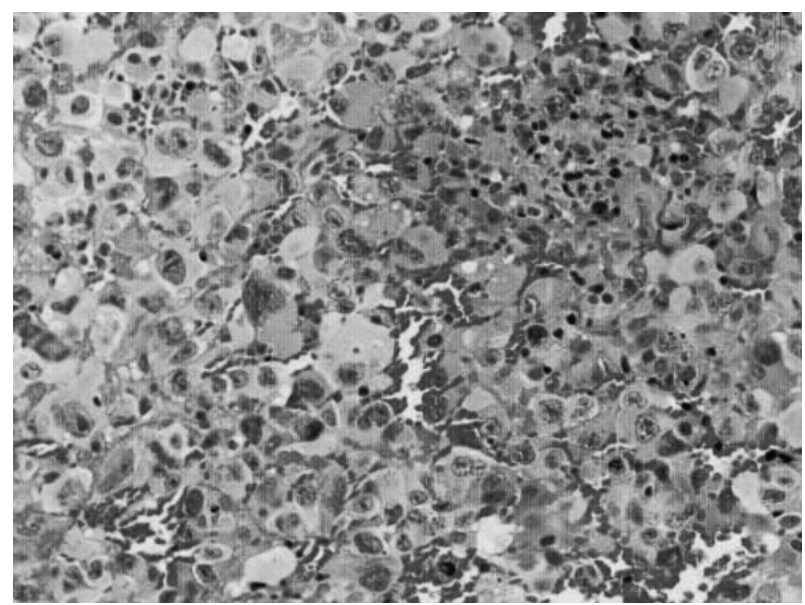

Fig. 3. Pathological findings of the brain around hematoma in the left frontal lobe. The section consisited of a diffuse proliferation of atypical giant cells. No sarcomatoid component was seen.

sis from lung GCC. Since the patient failed to recover consciousness we recommended against performing adjuvant therapy. The follow-up CT series revealed remarkable enlargement of the hematomas, especially in the left cerebellum. Unfortunately, one month after admission she died of progressive prostration.

\section{DISCUSSION}

This is a case of brain metastasis from a rare pulmonary GCC. Pulmonary GCC in particular is generally regarded as a distinct entity based on its unusual histologic features and its extremely aggressive clinical course [3,4]. This case demonstrated multiple intracerebral hemorrhages with remarkable enlargement in a short period. There are some reports of intratumoral 
hemorrhage associated with brain metastasis from lung cancer. However, simultaneous hemorrhage of multiple metastatic lesions is uncommon. In general we should consider brain tumor, along with hypertension, trauma, amyloid angiopathy, hemorrhagic infarction, hematologic disorder, liver disease and drugs, as possible causes of multiple intracerebral hemorrhage. Among metastatic brain tumors, malignant melanomas as well as choriocarcinoma and renal cell carcinomas frequently develop significant hemorrhages. With regard to etiology, the causes of intratumoral hemorrhage are often considered to be endothelial proliferation with vascular obliteration, vessel compression and distortion due to rapid tumor growth, vessel necrosis, invasion of vessel walls by the tumor, and increased venous pressure associated with increased intracranial pressure [5,6]. In some cases, hypertension and coagulopathy are thought to be risk factors of intratumoral hemorrhage. Similar mechanisms, i.e. aggressive invasion and neovascularity of GCC, are likely to be associated with the multiple intracerebral hemorrhages in this case. Moreover, in spite of bed rest, medication with hemostatic drugs and the absence of high blood pressure or abnormalities in the coagulating system, a remarkable enlargement of the hematomas occurred in a short period.

Intracerebral multiple metastases were detected with hemorrhage, but the primary lung GCC was detected without bleeding in our case. Shoji et al. [7] reported a case of small intestinal metastases with bleeding 6 days prior to the discovery of bleeding from a primary lung GCC. We speculate that both the primary and metastatic lesions have an equally strong tendency to bleed. Lung carcinoma often metastasizes to the brain. Although adjuvant therapies such as radiation and chemotherapy are often performed in addition to surgical resection, the prognosis is still poor. Shoji et al. [7] reported a rare case of stage IV (without brain metastasis) pulmonary GCC who has survived long-term after undergoing aggressive surgical treatment and chemotherapy. However chemotherapy for brain metastasis from pulmonary GCC is unlikely to be effective due to problems with drug delivery. We should consider metastatic brain tumor as a possible cause in cases of multiple intracerebral hemorrhage. In this case, we were able to easily detect lung carcinoma on chest CT. However, because metastatic lesions from GCC have a tendency to bleed [7], intracerebral hemorrhage might be discovered before the detection of lung carcinoma in some cases.

\section{REFERENCES}

1. Travis WD, Colby TV, Corrin B, Shimosato Y, and Brambilia E. World Health Organization International Histological Classification of Tumors. Histological typing of Lung and Pleural Tumors. 3rd edition. Berlin: Springer Verlag; 1999.

2. Brambilia E, Travis WD, Colby TV, Corrin B, and Shimosato Y. The new World Health Organization classification of lung tumors. Eur Respir 2001; 18:1059-1068.

3. Shin MS, Jackson LK, Shelton, Jr RW, and Greene RE. Giant cell carcinoma of the lung. Clinical and roentgenographic manifestations. Chest 1986; 89:366-369.

4. Zhao ZL, Song N, Huang QY, Liu YP, and Zhao HR. Clinicopathologic features of lung pleomorphic (spindle/ giant cell) carcinoma-A report of 17 cases. Ai Zheng 2007; 26:183-188.

5. Hirano A, and Matsui T. Vascular structures in brain tumors. Human Pathol 1975; 6:611-621.

6. Kondziolka D, Bernstein M, Resch L, Tator CH, Fleming JF et al. Significance of hemorrhage into brain tumors: clinicopathological study. J Neurosurg 1987; 67:852-857.

7. Shoji F, Maruyama R, Okamoto T, Ikeda J, Nakamura T et al. Long-term survival after an aggressive surgical resection and chemotherapy for stage IV pulmonary giant cell carcinoma. World Journal of Surgical Oncology 2005; $3: 32$. 